\title{
Deployment and Mobility for Animal Social Life Monitoring Based on Preferential Attachment
}

\author{
Mustafa İlhan Akbaş*, Matthias R. Brust*, Carlos H.C. Ribeiro ${ }^{\dagger}$, and Damla Turgut* \\ * Department of Electrical Engineering and Computer Science \\ University of Central Florida, Orlando FL \\ Email: \{miakbas,mbrust,turgut\}@eecs.ucf.edu \\ ${ }^{\dagger}$ Computer Science Division \\ Technological Institute of Aeronautics, Brazil \\ Email: carlos@ita.br
}

\begin{abstract}
The effort and time spent in wild life monitoring increases with the area and the number of objects to observe. Deployment of wireless sensor nodes enables a scalable sampling method and fine granularity of data collection. When we include resource rich actor nodes the data collection and evaluation are further optimized. However realistic mobility data is missing for various animal species to develop prototypes of wireless network based monitoring systems.

In this paper, the problem of the absence of realistic data is considered from a social network perspective. Node deployment and mobility algorithms are provided to model a complete system of an animal swarm to be used for animal social life monitoring. A novel spatial cut-off preferential attachment model and center of mass concept are used and extended for the models according to the characteristics of the animal swarms. In the application scenario of a gorilla swarm, each gorilla is equipped with a sensor node for the monitoring of the social system. The local interaction patterns among the mobile nodes are also monitored throughout the network life time to observe the social interactions among animals and to determine the role of each animal in the society. The performance of the monitoring protocol and the applicability of the deployment and mobility models are presented through extensive simulations.
\end{abstract}

\section{INTRODUCTION}

The continuous monitoring of animal groups in their natural habitat and captureing their distinctive behaviors involves technically sophisticated planning, correct installation, successful execution, reliable data collection, and reasonable data interpretation. Due to the complexity of all these relevant processes, the conventional approaches for wild life monitoring have to deal with a number of challenges [1]. Most of the conventional methods used so far are highly invasive on the environment and animals under study, which is difficult, in particular when periodical resampling is needed. In addition, it is crucial to decide on the correct sampling method that delivers the demanded data for answering the research questions. Monitoring also requires an appropriate design of the sampling process to guarantee accurate and precise measurements, which are challenging especially when monitoring an animal population changing its habitat frequently. Additionally, the extra effort to plan and execute a monitoring project tremendously increases with the area size and number of objects (animals) under investigation as well as the period of observation.

Making use of the most recent advances in wireless networks can overcome many of these drawbacks and challenges. Wireless sensor and actor networks (WSANs) [2] consist of tiny sensor nodes sensing events in the environment, and a limited number of more powerful mobile actors, which can actively collect information from the sensor nodes, process this information and react to the events.

A sensor node has limited data processing capability, transmission rate, energy, and memory. Actors, on the other hand, possess increased computation capabilities and wider communication ranges while equipped with long lasting batteries and larger memory compared to sensor nodes. Improvements in size, weight, energy and sensing capabilities of sensor nodes as well as the self-organizing aspects of the participating nodes make WSANs suitable for wild life monitoring, in particular for wild social life.

For various animal species, realistic movement data is missing, yet needed for simulation studies to test such elaborated wireless network based monitoring systems. Consequently, it is crucial to design a proper mobility model derived from the expected mobility patterns from the animal group under observation.

In this paper, we describe data collection and evaluation algorithms to provide a complete system of animal monitoring model for network deployment and mobility within a WSAN. More specifically, we consider the modelling and monitoring of the social life of animal swarms. We approach the problem of the absence of realistic data to model the movement patterns from a social network perspective. The preferential attachment idea is used for node deployment and mobility. The existing preferential attachment models do not have any limitation in the node degree, which violets known attachment limitations for different members in a group. Therefore a contribution of this paper is to provide a cut-off preferential attachment model based on spatial relationship of the nodes.

We choose gorillas to model in this paper. Such animals 
build and live in a hierarchal social network and a group of gorillas belonging to the same social network is called a "troop". Many characteristics of the gorilla social life are already known [3]. However, the lifespan of gorillas can vary between 30 to 50 years with gorillas reproducing and leaving the troop or constantly moving with the troop to new habitats, many more aspects of their social life remain unknown. The gorilla troop has a strict social organization, and it remains a challenge for scientists to find out how they interact and how their affiliations might change without disturbing the troop due to the presence of cameras or observers.

By equipping the gorillas with sensors, a WSAN can capture essential characteristics of each gorilla such as its interactions with its environment or troop members, its movement, speed, and so on. The proposed protocols use the collection of the spatial-temporal relationships to decide on the role of each animal in the society automatically and locally.

This paper is an initial work and provides a base model for deployment and mobility of gorilla troops. The approach provides a model for the development of monitoring applications. The model is verified by known social features of the gorilla society.

The remainder of this paper is organized as follows. Related work is presented in Section 2. The system model is presented in Section 3. The detailed descriptions of network deployment and node mobility are provided in Section 4 . The data collection and directory formation are given in Section 5 . We present the simulation results in Section 6 and finally conclude in Section 7.

\section{RELATED WORK}

\section{A. Sensor deployment}

There are various studies on the deployment of sensor nodes for wild life animal monitoring and tracking. Environmental scientists and zoologists have been increasingly using these technologies to collect data from wild terrestrial areas and transmit them to the remote databases [4]. In some of these applications, the sensor nodes are attached to the animals, forming an ad hoc wireless network of mobile nodes [5].

ZebraNet [6] is one of the initial examples of such studies, in which the animals carry custom tracking collars with GPS capability and form a mobile sensor network across a large area. The collars include sensor nodes with global positioning system (GPS) capability, and they form a WSN to monitor the area and record the data. Wark et al. [5] apply sensor network technology to farming and their approach also includes collars worn by animals. This system utilizes both static and mobile nodes measuring the state of a complex, dynamic system comprising climate, soil, pasture, and animals. Naumowicz et al. [7] deployed a WSN on Skomer Island, Wales to improve the investigation of the behavior and spatial ecology of the Manx Shearwater seabirds. The system informs the scientists with high resolution data about the arrival and departure of the birds and the environmental parameters such as temperature or humidity. Handcock et al. [8] uses a large cattle enterprise to demonstrate the potential for combining GPS collars and satellite images in a WSN. The implemented WSN is used to monitor behavioral preferences and social behavior of cattles in Northern Australia. There are also sensor network applications where the sensor nodes are implanted within the animal body in order to monitor the functioning of the whole body or a particular organ of the animal (see [9]).

\section{B. Group and swarm mobility models}

Gathering real field data is challenging for studies on animal behavior. For instance, Fossey [10] conducted the initial studies on the home ranges of the mountain gorillas and their social interactions. Fossey's observations include hand drawn maps of mountain gorilla ranging paths that form the basis for movement patterns of the gorilla troops. The gorilla behaviors and social interactions in troops are analyzed in a few studies [3], [11]. Due to the absence of public domain real data in this area, models for the generation of movement patterns must be developed and used. A variety of mobility models have been proposed for simulations of groups and swarms.

The Reference Point Group Mobility (RPGM) [12] by Hong et al. describes mobility coherence in the movement of a mobile host, i.e. hosts at different positions head towards the same target. In RPGM each group has an own logical center and similar to the concept of center of mass, the center's motion defines the entire group's motion behavior. The node deployment in RPGM is usually uniformly at random, but any node deployment can be used to approach the reference point. Nodes in RPGM have their own random movement in addition to the group motion.

In the Virtual Track model (VT model) [13] by Zhou et al., nodes follow so called "switch stations" that are deployed in the map creating virtual tracks. Group nodes are distributed along the virtual tracks and the individual nodes are deployed in the whole area. The switch stations have features allowing the nodes to split into several groups after leaving the switch station. These aspects can be often found in the mobility of animal swarms like birds or gorilla troops, which split when a new leader founds a new troop.

Musolesi et al. [14] approach the problem of the absence of realistic data to model movement patterns from a social perspective. Their model groups collection of nodes together based on social relationships among the individuals. The groups are mapped to a topographical space, including the strength of social ties. A node belonging to a group moves inside the corresponding group area towards a goal using the Random Waypoint model. Groups also move towards randomly chosen goals with random speeds. As in the model of Hong et al. [12], Musolesi et al. [14] also permits changes in the group affiliation based on a particular parameter (sociability factor).

The deployment and mobility models introduced in this paper use the "preferential attachment" concept, which is implemented by Borrel et al. [15] for designing the mobility model called Pragma. The preferential attachment was introduced by Barabási and Albert [16] to explain a common 
property of many large networks, according to which the vertex connectivities follow a scale-free power-law distribution. Pragma assumes preferential attachment to centers of interest, considering that "individuals" move towards "attractors", which appear and disappear. Thus, the model describes independent nodes that exhibit a collective behavior. The authors show that Pragma achieves a scale-free spatial distribution in population growth.

\section{System MODEL}

\section{A. Network model}

The animals in the network are equipped with sensor nodes except the troop leaders, which are equipped with the actors. On some rare points in the habitat, additional actors are installed at accessible points to collect data from the actor network when they are in transmission range. These actors form the backbone of the network and work as the gateways for the mobile network. Fig. 1 shows an example for such a WSAN implemented for a gorilla society.

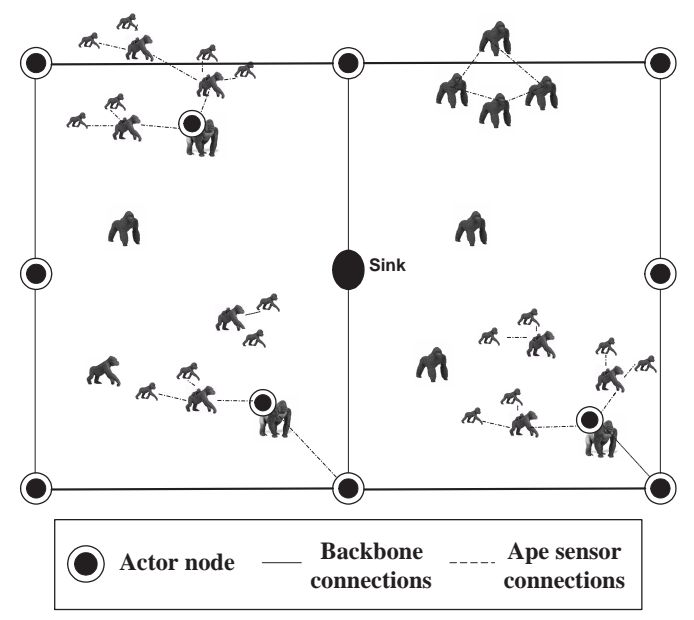

Figure 1. Application scenario of gorillas.

All communication and packet exchange in the network occur only locally. When communicating with the sensor nodes and creating the affiliation areas, the actors use the same transmission range, $r_{s}$, as the sensor nodes use when they exchange packets with their neighboring sensor nodes. Each actor encodes the transmitted packets with its $I D$ and weight $k$ to update the weight of the sensor nodes.

\section{B. Gorilla social structure}

Gorillas live in socially organized troops. Animals in the troop have different roles depending on the gender, age, strength and affinity. For instance the dominant, adult male, who leads the group in daily travels is called the silverback and has exclusive breeding rights to the females. Generally, there is one leader silverback in each troop. Differently from other primates, adult females do not interact with each other and usually compete to groom and stay close to the silverback. There are three or four adult females in each troop in general. Blackbacks are the juvenile males and they usually do not

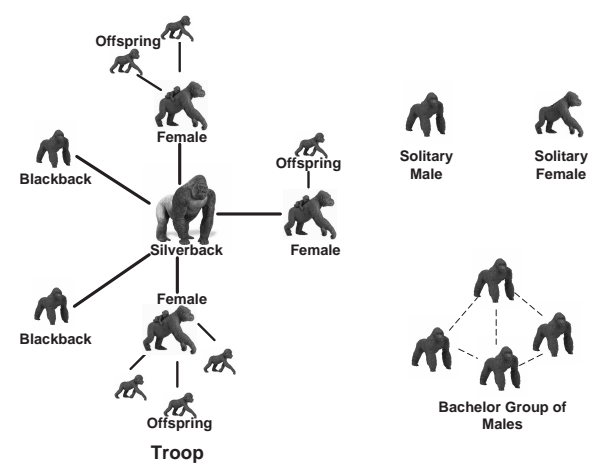

Figure 2. Gorilla social structure.

get close to the silverback since the silverback can see them as threats to its authority. Maturing males usually leave their family groups to establish either their own band or to join a bachelor group. Juvenile females stay closer to the troop compared to blackbacks and may change family groups multiple times until having an offspring. Newborns have strong relationships with their mothers and stay very close for the next three to four years. The roles of the individual animals in the society build up a hierarchical structure, which is shown in Fig. 2 for our application scenario.

Gorillas have complex social lives with families, affections, and politics of their own. The behaviors of gorilla societies and the social structure itself shows great variety. Therefore the information and assumptions used in our approach follow the general guidelines, considering well known facts about gorillas. For instance they are not territorial, they form a new nest every night at the location where they reach by their walk during the day. These known properties are critical inputs for the introduced deployment and mobility models. Different animal swarms can exhibit different social structures. The models presented in this paper provide a base model that can be adapted accordingly.

\section{DEPLOYMENT AND MOBILITY}

\section{A. Network deployment}

The distribution of nodes in the environment is one of the critical factors attempting to model the structure of a society. In this paper, we introduce two approaches for initial network deployment. The first approach is based on the preferential attachment method. The second approach uses the center of mass concept combined with the information gathered from the literature on animal swarm.

1) Preferential attachment approach: The result of the deployment of nodes according to this approach is a "scalefree" network. In scale-free networks, the distribution of the number of node connections follows a power law distribution. The frequency of an event has a power law distribution when it varies as a power of some attribute of that event.

The network deployment is initialized by positioning two sensor nodes in the area such that they are in transmission range of each other. Let $G_{n}$ be the resulting graph of the 
network when the $n^{t h}$ node $\left(v_{n}\right)$ is added to the existing nodes in the network. When obtaining $G_{n}$ from $G_{n-1}$ according to preferential atatachment, the probability of adding a link from $v_{n}$ to $v_{i}, P(i)$, is proportional to the degree $\left(d_{i}\right)$ of $v_{i}$.

The deployment model of our protocol extends the preferential attachment to be used for animal societies. The social structure of most animals is clustered such that there are subgroups in the entire animal group. For example the silverback in a gorilla society is accompanied by one to four females, which are surrounded by their offspring almost all the time and these youngsters generally do not exceed three. A new parameter, called "maximum degree" $\left(d_{\max }\right)$, is introduced to include these properties of the animal social structures.

The network is deployed according to the preferential attachment until one of the nodes has the "maximum degree". When a node has a degree of $d_{\max }$, it must not be eligible as a new deployed node for others to get connected. Therefore, the $P(i)$ value of a node depends not only on its degree but also on the $d_{\max }$ defined for the animal group. After the node reaches $d_{\text {max }}$, the node's $P(i)$ is reduced to the value, $P_{c}$, which depends on the characteristics of the animal society. Hence the probability of adding a link from $v_{n}$ to $v_{i}$ is as follows:

$$
P(i)=\left\{\begin{array}{cl}
\frac{d_{n}}{\sum_{i=1}^{N} d_{i}} & \text { if } d_{i}<d_{\max } \\
P_{c} & \text { if } d_{i} \geq d_{\max }
\end{array}\right.
$$

where $d_{i}$ is the degree of the node $i$. The decision process on the deployment of a new node joining the network is given in Algorithm 1.

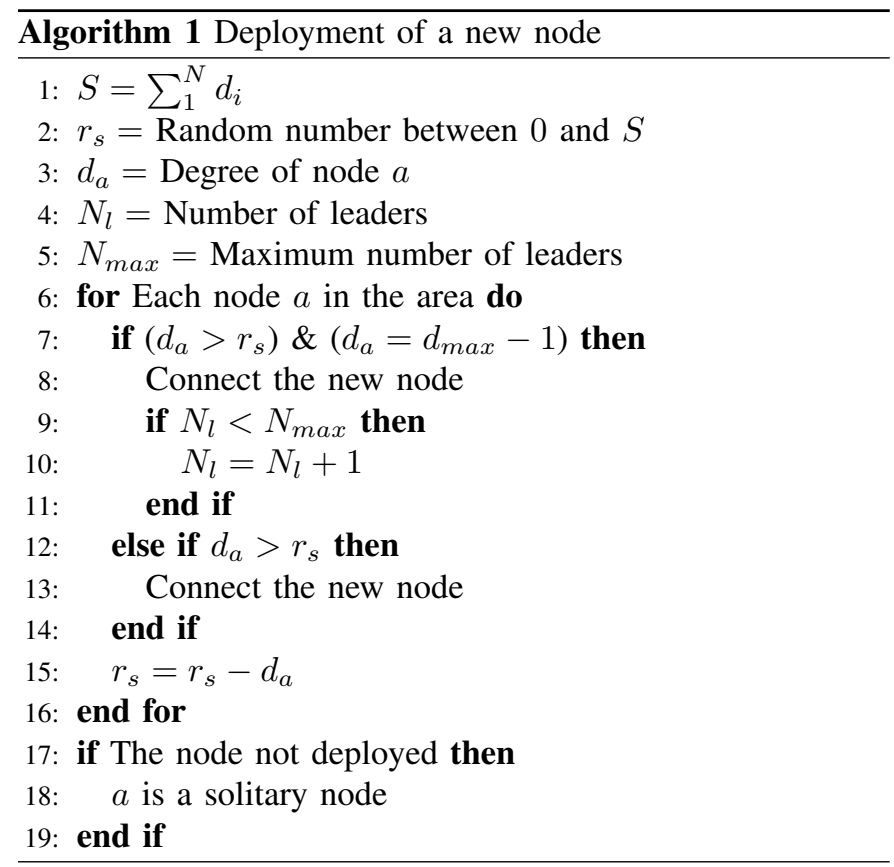

In our scenario, each gorilla in the network can be connected to only one silverback and there cannot be a link between two silverbacks. Hence the links against these rules are removed.

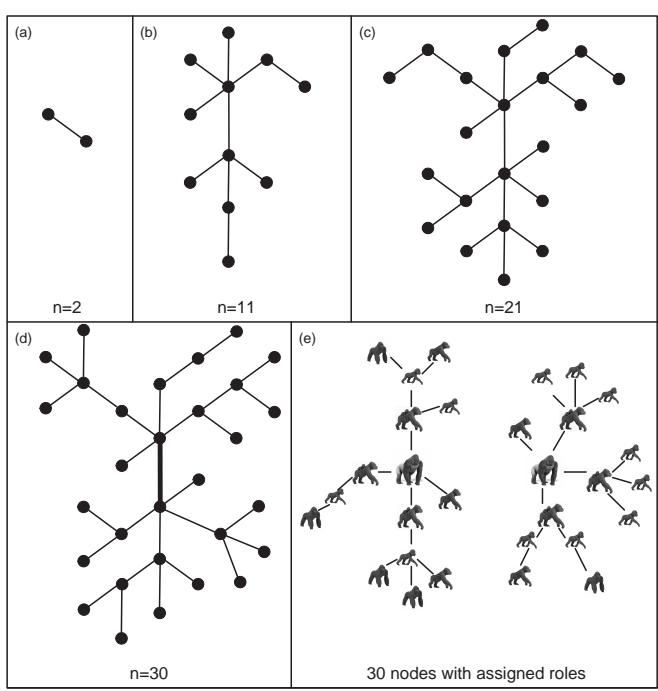

Figure 3. Deployment of nodes by preferential attachment based method.

The preferential attachment based deployment method can be extended by adding more species-specific features. For instance, as a gorilla troop moves in its environment, the leader of the troop avoids close encounters with other troops. Therefore some of the links are removed after the nodes are deployed and the roles are assigned.

Fig. 3 shows an example sequence for network deployment. In this scenario, $d_{\max }$ and $P_{c}$ are taken as five and zero respectively. Hence the nodes having a degree of $d_{\max }$ during deployment are no longer candidates for new nodes to get connected. In Fig. 3 (e), the roles assigned to the nodes are shown. There are two silverbacks in the society and it can be seen in the previous frame that they are connected by a link (plotted thicker). This link is removed after the roles are assigned according to the gorilla-specific rule stating that there cannot be a link between two silverbacks.

2) Center of mass based approach: In this approach, the nodes are distributed in the area according to a predefined structure. This structure depends on the type of the species under observation and it is defined by the ratio among the specific roles in the society.

The center of mass concept of physics is used in accordance with the hierarchy in the animal society. The animal society is divided into subgroups such that each subgroup's center of mass is their leader from the higher level group. For instance, the breeding females form a group and their center of mass is taken as the silverback. Similarly, a mother gorilla is chosen as the center of mass for its offspring. This method is applicable to and modifiable for any animal swarm. According to the center of mass approach, the coordinates of the nodes around the leader must satisfy the following equations:

$$
x_{s}=\sum_{i=1}^{N} \frac{x_{i}}{N} \quad y_{s}=\sum_{i=1}^{N} \frac{y_{i}}{N}
$$

where $\left(x_{s}, y_{s}\right)$ is the position of the leader and $N$ is the 
number of nodes in that subgroup. This method is extendable for different scenarios. For instance if a mother has four infants, the possible positions of these nodes can be limited depending on their ages so that two of them will be very close to the mother whereas the others keep a larger distance from her.

\section{B. Mobility model}

Lévy walk is observed as the mobility model in most of the animal foraging patterns, such as jackals [17] or spider monkeys [18] and it is recognized as an optimal way to find randomly dispersed objects [19]. It is a random walk with step-lengths distributed according to a heavy-tailed probability distribution. Lévy walks are Markov processes and after a large number of steps, the distance from the origin of the random walk tends to be reach stable distribution. Lévy distribution is the Fourier transformation of the moving distance of a single random walk and Rhee et al. [20] gives its PDF as follows:

$$
f_{z, \alpha}(x)=\frac{1}{2 \pi} \int_{-\infty}^{\infty} e^{-i z t} \phi(t) d t
$$

where $\phi(t)=e^{-|C t|^{\alpha}}$ and $C$ is a constant.

The distribution can be approximated by a power law of the form $y=x^{-\alpha}$ where $0<\alpha<2$. Each step in Lévy walk can be expressed by a tuple $L=\left(1, \theta, \Delta t_{f}, \Delta t_{p}\right) . \Delta t_{f}$ indicates the duration and it is chosen for each walk from a probability distribution $P(l) . \Delta t_{p}$ specifies pause time at the end of a walk and $\theta$ is the random direction taken by a node. A Lévy walk contains many short walks and a small number of long walks. The resulting pattern of depends highly on the value of $\alpha$ used in the system. As $\alpha$ becomes greater, the number of short walks increases.

One of the most common behaviors observed in diverse species is that they live in groups and follow the leaders of their groups. Scientists have various explanations such as the increased safety or breeding opportunities in a group for these behaviors. In nature, the silverback makes the decisions for the selection of paths that the troop follows. The silverback role in a troop is assigned to a node in the network deployment phase of our scenario. Similarly, the mobility model of the troop depends on the movements of the silverback in both of the mobility models introduced. The silverback moves according to Lévy walk and the movements of other members of the troop are directed mainly by the silverback's path. This path is used as the main input when positioning the nodes at each time instant. Two additional methods supplementary to Lévy walk are used to determine the mobility of each node.

1) Preferential attachment based method: This method complements the preferential attachment based deployment. After the nodes are deployed, based on the moves of the nodes leading their corresponding groups, the moving directions of the other nodes are probabilistically decided. The probability is defined according to the nodes' roles and levels of proximity to their group leaders. Since the deployment attributes of nodes such as their assigned degrees and roles determine their initial positions, these attributes and therefore the preferential

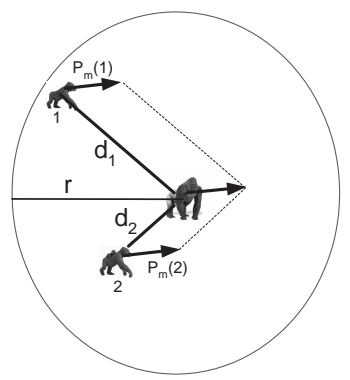

Figure 4. An example showing moving probabilities for females.

attachment method also affect the decisions on the movements of the nodes.

Each animal moves based on the mobility of its neighbor with highest degree, its distance to this neighbor and this neighbor's moving direction. This is a characteristic of the mobility model, which matches with the hierarchical structure of animal swarms. Hence the mobility of the offspring is based on the mobility of its mother, whose movement in turn depends on the mobility of the silverback. This structure also provides consistency with the approach of utilizing Lévy walk for the silverback in order to obtain a Lévy walk pattern for the whole troop.

The animals, which are close to their leaders, tend to stay close to the same position relative to such leaders, when the group moves. For instance, the newborns or infants are generally at most only a few steps away from their mothers. However juvenile animals forage in the environment and may walk in other directions. As they become adult animals, they may leave the troop. In order to include these characteristics, the nodes in close proximity of their leaders follow the leaders with a high probability, which decreases slowly as the distance of the node to the leader increases. Consequently, the model provides a Lévy walk pattern to the troop while providing possibilities for rare behaviors such as a bachelor male group formation. The probability $\left(P_{m}(i)\right)$ of a node to move in the same direction with its highest degree neighbor with transmission range $r_{a}$ is as follows:

$$
P_{m}(i)=\sqrt{\frac{r_{a}-d_{i}}{r_{a}}+c_{1}}+c_{2}
$$

where $d_{i}$ is the distance between the nodes. The constants $c_{1}$ and $c_{2}$ are included to provide the functionality of adjusting the probability calculation for different species types or network requirements. The Fig. 4 demonstrates an example of two females with their corresponding probabilities $\left(P_{m}(i)\right)$ to move in the same direction with the silverback, depending on their distances to the silverback. They move in any other random direction with probability $1-P_{m}(i)$.

The gorilla scenario includes an additional feature such that the nodes from different troops repel each other so that the troops are physically separated in the environment. 
2) Center of mass based method: This method is used to determine the positions of all animals at each time instant after the deployment of the nodes according to center of mass approach and the determination of the main path of the troop. A node's neighbors at hierarchically one lower level move in a coordinated fashion to have that node's position as their center of mass at all times.

This method allows a more controlled mobile network compared to the preferential attachment based method since the hierarchical structure of the system remains in its initial format throughout the network lifetime.

\section{DATA COLLECTION AND INTERPRETATION}

The data collected from animal monitoring systems are utilized for the interpretation of the social behavior of animals. Therefore the animal social structure must be utilized in the design of the data collection algorithms. The silverbacks become the natural clusterheads of their troops and the sensor nodes are affiliated with stationary and mobile actors in the network. According to a sensor node's affiliation and the cluster structure, the weight value of each sensor node is updated via local communication.

The network structure is formed and maintained by the state-transition rules defined only by local information. The nodes rapidly update their attributes as the network structure changes. A sensor node keeps only the maximum weight for each actor it receives packets from and it does not build a state or history of the whole network. When a sensor node receives a packet from an actor, the packet is retransmitted if its weight is less than the sensor node's weight for that actor. Otherwise, the sensor node drops the packet to avoid unnecessary traffic and energy consumption in the network. The weight $W_{A}(s)$ of each affiliation corresponds to $k$-hop distance of a node to an actor. The condition, in which a sensor node does not receive any weight updates, is defined as the loss of connection for the sensor node, which may refer to a solitary animal. In that case, the sensor node sets its hop value to the minimum value defined for the network. Then it operates only in listening mode and does not transmit any packets.

The spatial proximity is the main parameter to decide on the weights of the sensor nodes. The weight information for the nodes in a troop is collected at the corresponding mobile actor and transferred to the backbone whenever it is possible. The transmission range, time and cardinality are the parameters defining the rules for role assignment of the animals. The thresholds of these values can be generalized according to the animal group under observation while the rules are very specific for the social network of gorillas. Algorithm 2 gives the basic role determination process and the utilized rules.

\section{Simulation Study}

\section{A. Simulation environment}

We conducted extensive simulation experiment in the OPNET modeler [21]. The actors and sensor nodes are designed such that IEEE 802.11 is used as the underlying MAC layer. The transmission range of a sensor node is taken as 10 meters.

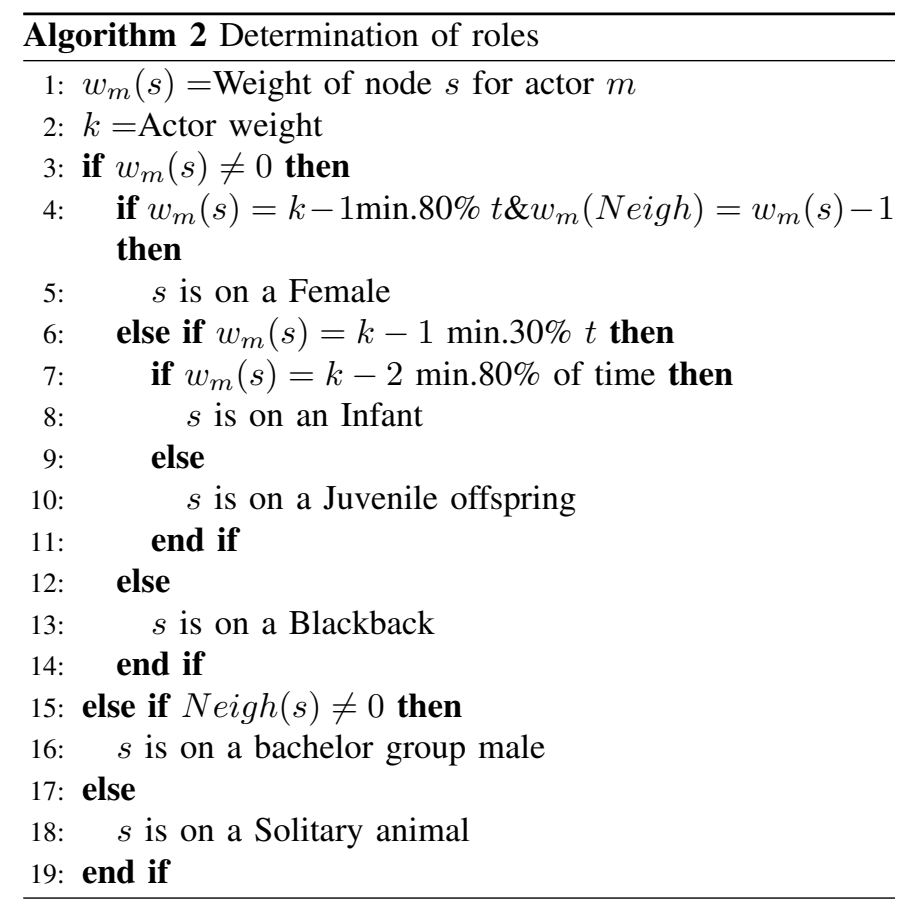

TABLE I

AVERAGE NUMBER OF ANIMALS FOR IN $1^{\text {st }}$ DEPLOYMENT SCENARIO

\begin{tabular}{|l|l|l|l|l|l|l|l|l|l|l|}
\hline Degree & 0 & 1 & 2 & 3 & 4 & 5 & 6 & 7 & 8 & 9 \\
\hline Pref. Att. & 0 & 7.03 & 1.83 & 0.93 & 0.46 & 0.30 & 0.21 & 0.18 & 0.13 & 0.09 \\
\hline PABD & 0.23 & 6.30 & 2.27 & 0.73 & 0.53 & 0.83 & 0 & 0 & 0 & 0 \\
\hline
\end{tabular}

The nodes in a network are positioned in the simulation area of $1750 \times 1750$ meters, which is sufficient considering the fact that gorillas are not fast moving animals; they move about 400 to 1000 meters everyday. A random waypoint mobility model is used in some simulations with speed uniformly distributed in $(3,8) \mathrm{km} / \mathrm{h}$ interval and pause time uniformly distributed in $(50,150)$ seconds interval. Such values of these metrics are determined according to the literature on gorillas [10]. The Lévy walk mobility model is used with $\alpha=1.9$ and $\beta=1.6$, which are the appropriate values for the foraging pattern of the considered gorilla troup based on the observed values in the nature (See [18]).

\section{B. Simulation results}

1) Deployment: Since the deployment model proposed in this paper is based on the preferential attachment model, it is tested against the original preferential attachment model in two sets of simulation studies.

In the first set of simulations, the deployment of only a single troop is considered. Gorilla troop populations in nature usually range from 2 to 12 members and the average troop size is 9. The total of 50 simulation runs are executed and in each simulation, 11 nodes are deployed in the area. In Table I, the average number of nodes for each number of connections are presented for the original preferential attachment and our preferential attachment based deployment model (PABD).

In the second set of experiments, there are 32 nodes 
TABLE II

AVERAGE NUMBER OF ANIMALS IN $2^{n d}$ DEPLOYMENT SCENARIO

\begin{tabular}{|l|l|l|l|l|l|l|l|l|l|l|}
\hline Degree & 0 & 1 & 2 & 3 & 4 & 5 & 6 & 7 & 8 & $>8$ \\
\hline Pref. Att. & 0 & 20.33 & 5.97 & 2.11 & 1.20 & 0.60 & 0.47 & 0.33 & 0.20 & 0.79 \\
\hline PABD & 0.66 & 17.45 & 5.17 & 2.93 & 2.03 & 3.03 & 0 & 0 & 0 & 0 \\
\hline
\end{tabular}

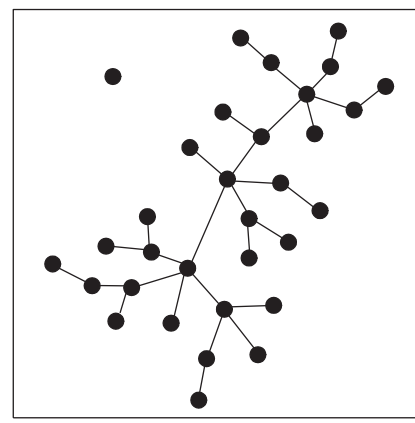

(a) Deployment by PABD.

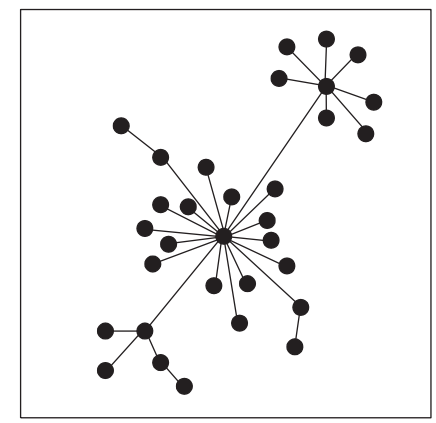

(b) Deployment by Pref. Attachment.
Figure 5. Node deployment by our protocol and by preferential attachment.

deployed in the area for each simulation. Table II shows the average number of nodes for each number of connections for the original preferential attachment and PABD.

The results given in I and II indicate that our deployment method produces gorilla troops with social relational properties similar to the troops in nature. The first result indicating this observation is that the degree distribution is more homogeneous compared to deployment by original preferential attachment while not any of the nodes exceeding a certain degree value. Another important result is that the solitary gorillas can be observed only in some scenarios of our deployment method.

One of the deployment cases by PABD, which has number of connections close to the average values, is presented in Fig. 5 to demonstrate the results visually. The figure shows that most of the nodes are directly connected to only a few nodes in the Preferential Attachment model, which is not a characteristic observed in gorilla society.

Fig. 6 shows the distributions of node degrees for both preferential attachment and our protocol in log scale. Even though the number of nodes is not very large, the power law linearization in a log scale can be observed. This behavior is not observed in PABD as a result of the modifications to the original preferential attachment method.

2) Roles: In the first set of experiments, nodes move according to the Random waypoint mobility pattern. Twenty simulation runs were executed with the same initial conditions and the average percentage of roles are determined by our role determination algorithm. In Fig. 7 (a), the percentages of the troop members over the simulation period are given. The percentages of the animals, which are not members of the troop, are given in Fig. 7 (b).

Fig. 7 shows that the number of solitary males increase with the simulation time and this role clearly becomes dominant in the society. The other roles have shares similar to each other, mostly depending on their initial positions and their directions. The solitary males in the nature walk alone in the habitat and they generally get affiliated with multiple troops over time, but can not become a member quickly. Hence this is an expected property for the society with random mobility with a starting condition in which most of the nodes are close to each other. Most of the nodes get departed from the troop as the time passes and the algorithms change their assigned roles to solitary males as they start to range alone in the area.

In the next set of experiments, the nodes move with Lévy walk with preferential attachment mobility pattern. Twenty simulation runs were executed and the average percentage of the roles are determined. The change in the percentage of the members and the non-members of the troop over simulation time are given in Fig. 8.

Fig. 8 shows that the percentage of each role fluctuates at the begining of the simulation similar to the initial experiment set with random mobility. This characteristic demonstrates that the role decision process requires a period of time to assign the correct roles to the animals. Another important result of the experiment shows that a Lévy walk with preferential attachment is an appropriate choice for mobility model, since the resulting average percentages of the roles match the structure of the real gorilla society network.

3) Rules: The introduced protocol for social role determination can be applied to any animal swarm by modifying its rules or by creating new metrics. The graphs in Fig. 9 show results for two different metrics chosen for the simulation scenarios with our preferential attachment based mobility (PABM), center of mass based mobility (CMBM) and random walk (RW). For the first case, it is assumed that the role distribution of the mobile society must be same as the role distribution in the stationary case. The metric for Fig. 9 (a) is the ratio of roles distributed in the mobile scenario to the roles distributed in the stationary case. For Fig. 9 (b), the used metric is the ratio of solitary animals to all animals in the society. Fig. 9 shows that CMBM gives results according to its design purpose such that the cluster structure remains same as the initial conditions. The results also demonstrate the probablistic nature of PABM since it differentiates from the initial deployment with a certain probability.

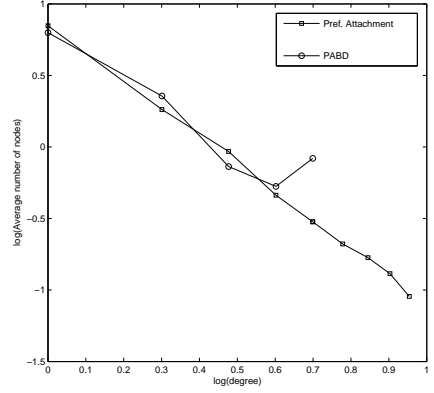

(a) Degree distribution for 11 nodes.

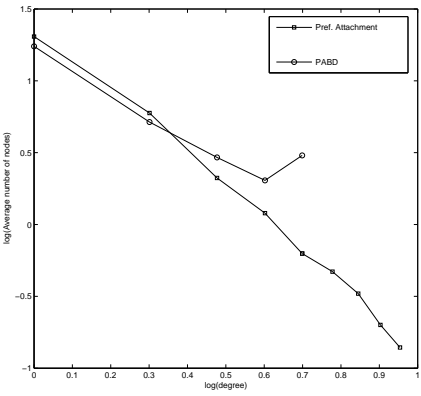

(b) Degree distribution for 32 nodes.
Figure 6. Degree distribution for PABD and by preferential attachment. 


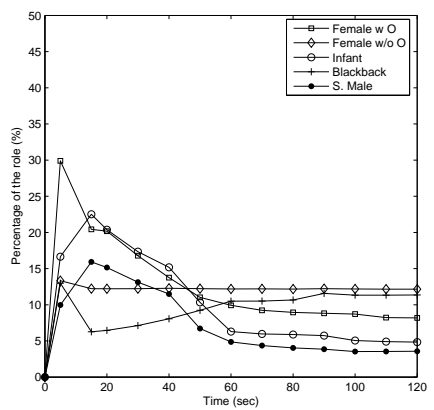

(a) Members of the troop.

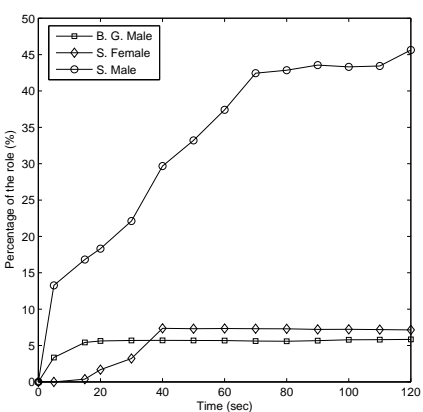

(b) Non-members of the troop.
Figure 7. Percentages of the roles vs. time in random walk.

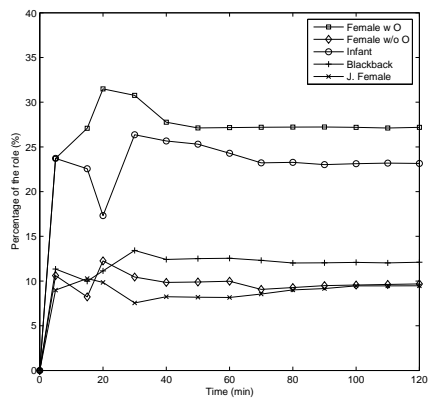

(a) Members of the troop.

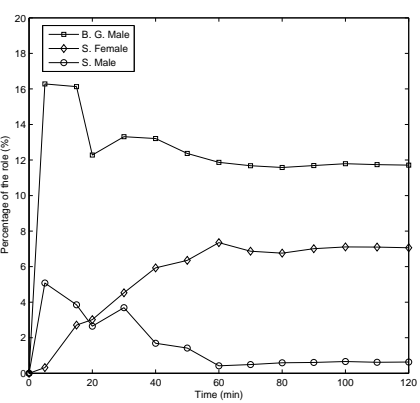

(b) Non-members of the troop.
Figure 8. Roles vs. time in Lévy walk with preferential attachments.

\section{CONCLUSION}

In this paper, we introduce node deployment and mobility algorithms to provide a complete system of animal swarm model for animal social life monitoring. These methods are based on the center of mass and preferential attachment concepts. The preferential attachment model is extended for the deployment and mobility models aaccording to the characteristics of the animal swarms. Both of the models are also extendible with properties specific to observed animal species. In the application scenario, each animal is equipped with a sensor node for the monitoring of the social system. In case of gorillas, it is shown how the data about the gorilla social structure in general aids in the design of an efficient protocol for capturing the social network of a troop. Simulation results

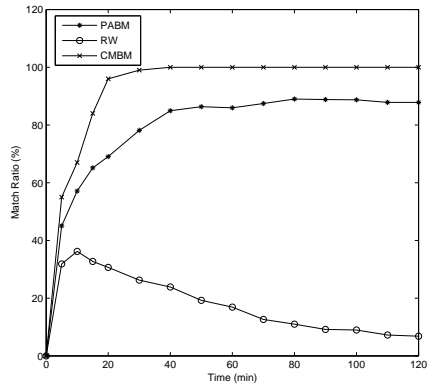

(a) All roles in the society.

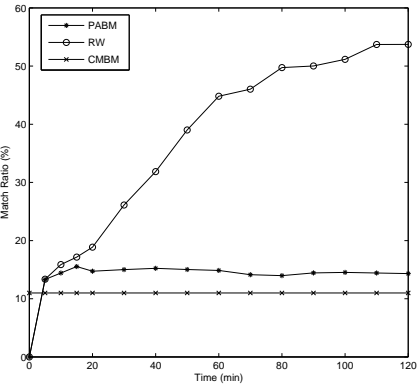

(b) Solitary animals in the society.
Figure 9. Behavior of the troop according to two different metrics. show that the outputs of preferential attachment based node deployment and mobility models match with the characteristics of the animal swarms under consideration. The social monitoring algorithm for deciding on the roles of gorillas uses the observed spatial-temporal interaction patterns. Future steps include generalization of the algorithms for other social systems and the verification of the results with field data.

\section{ACKNOWLEDGEMENT}

The authors would like to thank OPNET Technologies Inc. for supporting this research by providing OPNET Modeler software under OPNET University Program.

Matthias R. Brust gratefully acknowledges financial support by FAPESP (Fundação de Amparo á Pesquisa do Estado de São Paulo) under grant AP.PIPE 1 2010/51435-9 and RedeAlumni.

Carlos H. C. Ribeiro thanks CNPq for financial support.

\section{REFERENCES}

[1] M. L. Morrison, Wildlife restoration: techniques for habitat analysis and animal monitoring. Island Press, 2002.

[2] R. Verdone, D. Dardari, G. Mazzini, and A. Conti, Wireless Sensor and Actuator Networks: Technologies, Analysis and Design. Academic Press, 2008.

[3] M. M. Robbins and A. McNeilage, "Home range and frugivory patterns of mountain gorillas in bwindi impenetrable national park, uganda," International Journal of Primatology, vol. 24, no. 3, pp. 467-491, 2003.

[4] J. K. Hart and K. Martinez, "Environmental sensor networks: A revolution in the earth system science?" Earth-Science Reviews, vol. 78, no. 3-4, pp. 177-191, July 2006.

[5] T. Wark, P. Corke, P. Sikka, L. Klingbeil, Y. Guo, C. Crossman, P. Valencia, D. Swain, and G. Bishop-Hurley, "Transforming agriculture through pervasive wireless sensor networks," IEEE Pervasive Computing, vol. 6, pp. 50-57, April 2007.

[6] P. Juang, H. Oki, Y. Wang, M. Martonosi, L. S. Peh, and D. Rubenstein, "Energy-efficient computing for wildlife tracking: design tradeoffs and early experiences with zebranet," SIGPLAN Not., vol. 37, pp. 96-107, October 2002.

[7] T. Naumowicz, R. Freeman, A. Heil, M. Calsyn, E. Hellmich, A. Brändle, T. Guilford, and J. Schiller, "Autonomous monitoring of vulnerable habitats using a wireless sensor network," in Proc. of the Workshop on Real-World Wireless Sensor Networks. ACM, March 2008, pp. 51-55.

[8] R. N. Handcock, D. L. Swain, G. J. Bishop-Hurley, K. P. Patison, T. Wark, P. Valencia, P. Corke, and C. J. O'Neill, "Monitoring animal behaviour and environmental interactions using wireless sensor networks, gps collars and satellite remote sensing," Sensors, vol. 9, no. 5, pp. 3586-3603, May 2009.

[9] G. Z. Yang, Body Sensor Networks. Springer-Verlag New York, Inc., 2006.

[10] D. Fossey, "Observations on the home range of one group of mountain gorilla (gorilla gorilla beringei)," Animal Behaviour, vol. 22, pp. 568581, 1974.

[11] M. Boos, M. Kolbe, P. Kappeler, and E. T., Coordination in Human and Primate Groups. Springer-Verlag New York, Inc., 2011.

[12] X. Hong, M. Gerla, G. Pei, and C.-C. Chiang, "A group mobility model for ad hoc wireless networks," in Proc. of the 2nd ACM international workshop on Modeling, analysis and simulation of wireless and mobile systems, ser. MSWiM, August 1999, pp. 53-60.

[13] B. Zhou, K. Xu, and M. Gerla, "Group and swarm mobility models for ad hoc network scenarios using virtual tracks," in Proc. of IEEE MILCOM, November 2004, pp. 223-230.

[14] M. Musolesi, S. Hailes, and C. Mascolo, "An ad hoc mobility model founded on social network theory," in Proc. of MSWiM, October 2004, pp. 20-24.

[15] M. D. d. A. V. Borrel and S. Fdida, "A preferential attachment gathering mobility model," IEEE Communications Letters, vol. 9, no. 10, pp. 900902, October 2005. 
[16] A. L. Barabási and R. Albert, "Emergence of scaling in random networks," Science, vol. 286, no. 5439, pp. 509-512, October 1999.

[17] R. P. D. Atkinson, C. J. Rhodes, D. W. Macdonald, and R. M. Anderson, "Scale-free dynamics in the movement patterns of jackals," OIKOS, vol. 98, pp. 134-140, July 2002.

[18] J. Ramos-Fernandez, L. Morales, O. Miramontes, G. Cocho, H. Larralde, and B. Ayala-Orozco, "Lévy walk patterns in the foraging movements of spider monkeys (ateles geoffroyi)," Behavioural Ecology and Sociobiology, vol. 55, pp. 223-230, 2004

[19] G. M. Viswanathan, S. V. Buldyrev, S. Havlin, M. da Luz, E. P. Raposo, and H. E. Stanley, "Optimizing the success of random searches," Nature, vol. 401, pp. 911-914, October 1999.

[20] I. Rhee, M. Shin, S. Hong, K. Lee, and S. Chong, "On the Lévy-walk nature of human mobility," in Proc. of IEEE INFOCOM, April 2008, pp. 924-932.

[21] “Opnet modeler," http://www.opnet.com. 